\title{
Modelling a Conversational Agent with Complex Emotional Intelligence
}

\author{
Belainine Billal, Sadat Fatiha, Lounis Hakim \\ ${ }^{1}$ University of Quebec in Montreal \\ 2098 Rue Kimberley, Montréal, QC H3C 3P8 \\ Quebec, Canada \\ belainine.billal@hotmail.com
}

\begin{abstract}
Chatbots or conversational agents have enjoyed great popularity in recent years. They surprisingly perform sensitive tasks in modern societies. However, despite the fact that they offer help, support, and fellowship, there is a task that is not yet mastered: dealing with complex emotions and simulating human sensations. This research aims to design an architecture for an emotional conversation agent for long-text conversations (multi-turns). This agent is intended to work in areas where the analysis of users feelings plays a leading role. This work refers to natural language understanding and response generation.
\end{abstract}

\section{Introduction}

To create a conversational agent that can communicate with a human user, it is necessary to include in the machine capabilities, ability to perceive and express emotions. Existing studies show that considering effects and emotions in dialog systems can improve user satisfaction (Prendinger and Ishizuka 2005) and reduce the number of failures during dialogs. Some work has also been done to adjust dialog behaviors based on the emotional states of users (Huang et al. 2017) and to generate answers to user statements at the content and affect level (Skowron 2010). However, these studies, mainly inspired by work in psychology, are rule-based or limited to small-scale data.

Recently, large-scale data-driven neural models have put forward significantly the generation of open-domain conversations (Ritter, Cherry, and Dolan 2011; Vinyals and Le 2015). Most of these templates are designed to improve the quality of the conversation generation content. For the first time, a neural emotional agent model was proposed by (Zhou et al. 2017): ECM $^{1}$ which can generate appropriate responses based on six basic emotions (anger, disgust, joy, love, sadness, and other). This work is based on the detection and generation of a basic emotion according to the model proposed by (Ekman, Friesen, and Ellsworth 2013). In the same year, (Zhou and Wang 2017) offers MojiTalk and explore the idea of exploring emojis of Twitter data, which

Copyright (C) 2020, Association for the Advancement of Artificial Intelligence (www.aaai.org). All rights reserved.

${ }^{1}$ ECM-Emotional Chatting Machine are naturally tagged during the conversation. The authors assume that emoji transmit the underlying emotions of the sentence.

From our study of the existing work, we find that the factor of detection and generation of complex emotions has not been addressed in existing neural models for the generation of the conversation. In our research, we first focus on (1) equipping a chatbot with complex emotional intelligence, captured with the basic emotions, and emotional potential of a given event, then, 2) obtain knowledge about its behavior and then (3) ensure the exchange of emotional states between the agent and the user.

\section{Proposed Approach}

Our approach for creating an emotional agent capable of interpreting interlocutor's emotions and generating human interpretable responses is summarized by two main components. The whole process is illustrated in Figure 1:

(1) A unit of natural language understanding: This unit is responsible for the linguistic and emotional processing as well as the decomposition of sentences and the morphosyntactic form of words. It will generate vectors in the semantic space using the Word Embedding.

(2) A unit for generating answers: This unit is responsible for the recomposition of words in the form of syntactic and semantically valid sentences.

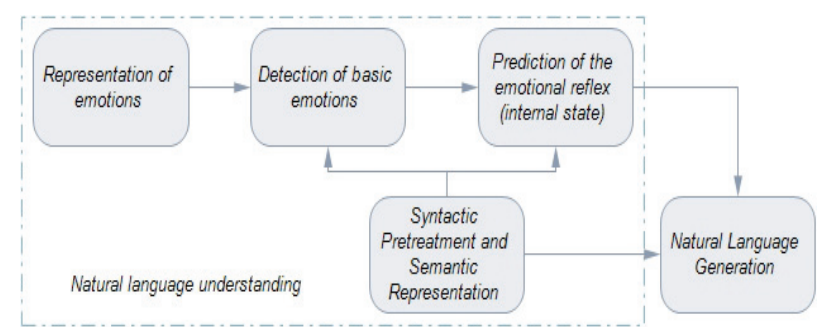

Figure 1: General presentation of the proposed method.

Our modeling of emotional states is based on the representation of emotional states in the form of vectors. For each emotional state there is a vector in a 4-dimensional space, 
each of their representing a pair of contradictory basic emotions (eg joy and sadness).

We propose to use the same basic emotions of the Plutchik model to define the dimensions of our base. Therefore, the number of dimensions of our basic emotion is 4 pairs of emotions and is formally defined by the base B = ((Joy, Sadness), (Trust, Disgust), (Fear, Anger), (Surprise, Anticipation)). So, any emotion can be realized from a combination of the other fundamental emotions that define our base B.

Each basic emotion of pleasure will be in the interval [0..1] and every basic emotion of displeasure will be in the interval [-1..0]. Thus, for the development of our solution, we resorted on Plutchik that addressed this problem of analogy with colors by proposing a dictionary of emotions similar to the dictionary of colors. A basic emotion is described by a vector that contains a single non-zero coefficient.

\section{Reading complex emotions}

Table 1 shows a representation of the primary complex emotions (according to the Plutchik model), and the combinations of 2 adjacent emotions separated by two emotions that make up the primary dyads. This table will be used as a transition matrix to detect the primary complex emotions.

Table 1: Combinations of 2 adjacent emotions separated by two emotions that make up the primary dyads in 4 dimensions.

\begin{tabular}{|l|l|r|r|r|}
\hline $\begin{array}{l}\text { Advanced Emotions Pri- } \\
\text { mary Dyad }\end{array}$ & Anticipation-Surprise & Joy-Sadness & Sympathy-Disgust & Fear-Anger \\
\hline \hline Optimism & 1 & 1 & 0 & 0 \\
\hline Love & 0 & 1 & 1 & 0 \\
\hline Submission & 0 & 0 & 1 & 1 \\
\hline apprehension & -1 & 0 & 0 & 1 \\
\hline Disappointment & -1 & -1 & 0 & 0 \\
\hline Remord & 0 & -1 & -1 & 0 \\
\hline Outrage & 0 & 0 & -1 & -1 \\
\hline Aggressiveness & 1 & 0 & 0 & -1 \\
\hline
\end{tabular}

Our problem can be seen as a multi-label learning problem where each of the four dimensions will be represented by a three-valued label $(-1,0,1)$. Our basic emotion detector will rely mainly on existing corpora such as DailyDialog ${ }^{2}$ which covers different domains.

\section{Predicting the emotional reflex (internal state)}

In order to respond well to the interlocutor, we should predict an internal emotion of our agent. Existing conversations in the corpus come from a personality that has its own emotion (internal state). This property requires us to learn the emotion of a single personality, and we should create a model that can predict the emotion of this personality based on the conversation of an interlocutor.

\section{Proposed model for long conversations}

The existing models in the literature do not make it possible to teach an agent the nature of the hierarchical sequence of the conversation. A conversation sequence is rotative, starting with a request followed by a response, and so on. However, existing models only allow learning queries alone, and

\footnotetext{
${ }^{2}$ http://yanran.li/dailydialog
}

for this reason, we propose an architecture that will allow agents to learn its answers.

In the proposed model for long conversation, the detected emotions can interfere in each stage of the conversation, memorizing the interaction in each step, based on the emotion detected during the human conversation, Then, in the last step, we plan to use the prediction of future emotion.

\section{Schedule and progress}

Table 2 describe my progress in September 2019, and the anticipation progress in date February 2020.

Table 2: Schedule and project progress

\begin{tabular}{|l|l|l|l|}
\hline Task & Research & $\begin{array}{l}\text { Progress } \\
\text { Sep 2019 }\end{array}$ & $\begin{array}{l}\text { Progress } \\
\text { Feb 2020 }\end{array}$ \\
\hline $\begin{array}{l}\text { 1: Model configuration } \\
\text { and data collection }\end{array}$ & $\begin{array}{l}\text { collection of corpus, thesauri and } \\
\text { pretreatments }\end{array}$ & $100 \%$ & $100 \%$ \\
\hline $\begin{array}{l}\text { 3: Model de- } \\
\text { sign/analysis for } \\
\text { complex emotions }\end{array}$ & $\begin{array}{l}\text { Development of the proposed } \\
\text { model for the detection of complex } \\
\text { emotions and the prediction of } \\
\text { emotional reflex }\end{array}$ & $80 \%$ & $100 \%$ \\
\hline $\begin{array}{l}\text { 4: Design/adjustment } \\
\text { of the complex emo- } \\
\text { tion detection model } \\
\text { and prediction of the } \\
\text { emotional reflex }\end{array}$ & $\begin{array}{l}\text { Application of the proposed method } \\
\text { and development of the prediction } \\
\text { system }\end{array}$ & $50 \%$ & $100 \%$ \\
\hline $\begin{array}{l}\text { 5: Model integration, } \\
\text { testing, validation and } \\
\text { comparisons }\end{array}$ & $\begin{array}{l}\text { Evaluation of the results obtained } \\
\text { and the beginning of the writing of } \\
\text { the thesis }\end{array}$ & $10 \%$ & $20 \%$ \\
\hline
\end{tabular}

\section{References}

Ekman, P.; Friesen, W. V.; and Ellsworth, P. 2013. Emotion in the human face: Guidelines for research and an integration of findings. Elsevier.

Huang, C.; Labetoulle, T.; Huang, T. K.; Chen, Y.; Chen, H.; Srivastava, V.; and Ku, L. 2017. Moodswipe: A soft keyboard that suggests messages based on user-specified emotions. CoRR abs/1707.07191.

Prendinger, H., and Ishizuka, M. 2005. The empathic companion: A character-based interface that addresses users' affective states. Applied Artificial Intelligence 19(3-4):267285.

Ritter, A.; Cherry, C.; and Dolan, W. B. 2011. Data-driven response generation in social media. In Proceedings of the Conference on Empirical Methods in Natural Language Processing, EMNLP'11, 583-593. Stroudsburg, PA, USA: Association for Computational Linguistics.

Skowron, M. 2010. Affect Listeners: Acquisition of Affective States by Means of Conversational Systems. Berlin, Heidelberg: Springer Berlin Heidelberg. 169-181.

Vinyals, O., and Le, Q. V. 2015. A neural conversational model. CoRR abs/1506.05869.

Zhou, X., and Wang, W. Y. 2017. Mojitalk: Generating emotional responses at scale. CoRR abs/1711.04090.

Zhou, H.; Huang, M.; Zhang, T.; Zhu, X.; and Liu, B. 2017. Emotional chatting machine: Emotional conversation generation with internal and external memory. CoRR abs/1704.01074. 